\title{
Ecosystem flux and biotic modification as drivers of metaecosystem dynamics
}

\author{
Romana Limberger,,${ }^{1}$ Julia Birtel,${ }^{1}$ Daniel d. S. Farias,${ }^{2}$ and Blake Matthews ${ }^{1,3}$ \\ ${ }^{1}$ Department of Aquatic Ecology, Eawag, Kastanienbaum, 6047 Switzerland \\ ${ }^{2}$ Programa de Pós-Graduação em Ciências Biológicas (Biodiversidade Neotropical), Universidade Federal do \\ Estado Rio de Janeiro, Rio de Janeiro, RJ 22290-240 Brazil
}

\begin{abstract}
The fluxes of energy, matter, and organisms are important structuring forces of metaecosystems. Such ecosystem fluxes likely interact with environmental heterogeneity and differentially affect the diversity of multiple communities. In an aquatic mesocosm experiment, we tested how ecosystem flux and patch heterogeneity affected the diversity of bacteria, phytoplankton, and zooplankton metacommunities, and the structure and functioning of metaecosystems. We built metaecosystems consisting of three mesocosms that were either connected by flux of living organisms, organic material, and nutrients (alive ecosystem flux) or only by flux of organic material and nutrients (dead ecosystem flux). The three patches of each metaecosystem were either homogeneous or heterogeneous in nutrient loading. We found that the three groups of organisms responded differently to our treatments: flux of living organisms increased bacterial diversity irrespective of nutrient heterogeneity, while flux effects on phytoplankton diversity depended on nutrient heterogeneity, potentially indicating source-sink effects. Although zooplankton diversity was largely unaffected by our manipulations, subtle changes of community composition in response to ecosystem flux had strong effects on lower trophic levels, highlighting the importance of indirect flux effects via alterations in trophic interactions. Furthermore, differential effects of communities on the mean and spatial variability of local abiotic environments influenced the development of metaecosystem heterogeneity through time. Despite identical nutrient loading at the scale of the metaecosystem, abiotic conditions diverged between homogeneous and heterogeneous metaecosystems. For example, concentrations in dissolved organic carbon (DOC) were higher in homogeneous than heterogeneous metaecosystems, possibly because of differential responses of the algal community to local environmental conditions. Similarly, we found that flux effects on organisms translated into effects on DOC concentrations at the patch level, suggesting that flux-mediated changes in abundances of species can alter abiotic conditions. Our study shows that the dynamics of biotic and abiotic compartments of spatially structured ecosystems are intricately linked, highlighting the importance of integrating metacommunity and metaecosystem perspectives.
\end{abstract}

Key words: bacteria; dispersal; DOC; ecosystem function; indirect effects; metacommunity; metaecosystem; phytoplankton; source-sink; zooplankton.

\section{INTRODUCTION}

Metacommunity and metaecosystem theory both posit that communities are structured by local abiotic and biotic conditions, as well as by dispersal and spatial heterogeneity of environmental conditions (Massol et al. 2011). While metacommunity theory focuses on how communities are shaped by dispersal (Leibold et al. 2004), metaecosystem theory also considers ecosystem flux, i.e., the flux of organic matter, in both detrital and living forms, and nutrients among ecosystem patches (Loreau et al. 2003, Massol et al. 2011). The two frameworks differ not only in the agents of patch connection, but also in the ecosystem properties they emphasize.

Manuscript received 3 July 2016; revised 11 December 2016; accepted 4 January 2017. Corresponding Editor: Bradley J. Cardinale.

${ }^{3}$ E-mail: blake.matthews@eawag.ch
Metacommunity theory focuses on diversity, while both biotic and abiotic ecosystem properties are modeled in metaecosystem theory (Massol et al. 2011), often, however, with a focus on biomass and nutrients (Gravel et al. 2010a, Marleau et al. 2010, 2014, Massol et al. 2011), rather than on diversity (but see Gravel et al. $2010 b$ ). In order to understand the dynamics of spatially structured ecosystems, it is important to integrate metacommunity and metaecosystem perspectives and quantify how diversity and composition of communities as well as abiotic conditions interactively respond to fluxes of organisms and material.

Both metacommunity and metaecosystem theory consider spatial heterogeneity and dispersal/flux as regional drivers of local community and ecosystem structure. The effects of dispersal on metacommunity structure depend on environmental heterogeneity (Amarasekare 2003, Leibold et al. 2004). For example, in heterogeneous 
metacommunities, dispersal can rescue species from competitive exclusion and thus maintain them in unfavorable sink habitats (source-sink dynamics; Mouquet and Loreau 2003), while rescue of species from stochastic or deterministic extinctions by dispersal is not contingent on environmental heterogeneity (Leibold et al. 2004). However, these expected outcomes from metacommunity theory can change if fluxes of organic matter and nutrients affect the dynamics of source and sink habitats, competition-colonization tradeoffs, and successional dynamics (Gravel et al. 2010a,b).

Metaecosystem theory recognizes that reciprocal interactions between communities and the abiotic environment can affect the dynamics of spatially structured ecosystems (Massol et al. 2011, Mouquet et al. 2013). There is compelling empirical evidence that both individual species and communities can have strong local effects on biotic and abiotic aspects of ecosystems (Chapin et al. 1997, Jones et al. 1997, Largaespada et al. 2012, Schmitz et al. 2014). For example, predators can alter local biogeochemical processes (Atwood et al. 2013, Schmitz et al. 2014), and herbivores can alter nutrient availability (Bardgett and Wardle 2003, Cherif and Loreau 2013). In addition, changes in the community composition of primary producers and decomposers can affect abiotic ecosystem properties (Giroldo and Vieira 2005, Gessner et al. 2010, Logue et al. 2016). Such localscale interactions between organisms and their environment can both affect and be affected by processes at the regional scale. For example, ecosystems that harbor species performing critical ecosystem functions can disproportionately affect regional dynamics, in particular when such keystone ecosystems influence the amount or composition of fluxes between ecosystems (Mouquet et al. 2013). Also, dispersal might influence reciprocal interactions between community dynamics and abiotic properties of metaecosystems, when dispersal affects species that have strong ecosystem effects.

To understand the dynamics of spatially structured ecosystems, we need comparative and experimental studies that test predictions arising from the integration of metacommunity and metaecosystem theory. Metacommunity studies have recently expanded so as to consider multiple interacting communities across multiple trophic levels (Verreydt et al. 2012, Declerck et al. 2013). Both comparative and experimental studies have observed that dispersal has differential effects on communities (Beisner et al. 2006, De Bie et al. 2012, Verreydt et al. 2012). Such patterns are typically attributed to differences in dispersal abilities (De Bie et al. 2012), but could also be influenced by variation in the relative importance of deterministic (i.e., niche-based) processes vs. stochastic processes (i.e., random changes in abundances) (Östman et al. 2010, Farjalla et al. 2012, Soininen et al. 2013). Importantly, the effects of dispersal can interact with both deterministic and stochastic processes, for example by influencing either ecological drift or deterministic outcomes of competitive or trophic interactions
(Vellend 2010). Because organisms can both respond to and modify the environment, the effects of dispersal on diversity and community composition may translate into effects on abiotic ecosystem properties.

In a large-scale aquatic metaecosystem experiment, we tested for interactive effects of environmental heterogeneity and ecosystem fluxes on (1) the diversity of bacteria, phytoplankton, and zooplankton communities, and (2) on the abiotic conditions and functions of aquatic ecosystems. Metaecosystems were either homogeneous or heterogeneous in nutrient loading and either connected by flux of living organisms, detritus and nutrients or connected only by flux of detritus and nutrients. With this experimental design, we could make predictions that build on core ideas of metacommunity and metaecosystem theory. From metacommunity theory (Mouquet and Loreau 2003, Leibold et al. 2004), we expected that environmental heterogeneity would increase regional diversity and dissimilarity in metacommunities governed by deterministic, niche-based processes, but would have no effect in metacommunities governed by stochastic processes. We predicted that flux of living organisms would increase local diversity irrespective of environmental heterogeneity in metacommunities governed by stochastic processes, while in metacommunities structured by niche-based processes live flux would increase local diversity only in heterogeneous metaecosystems. Building on the concept of keystone ecosystems from metaecosystem theory (Mouquet et al. 2013), we predicted that differential responses of organisms to local environmental conditions coupled with species-specific modification of the environment would result in uneven contributions of local ecosystems to metaecosystem dynamics. Specifically, we hypothesized that homogeneous and heterogeneous metaecosystems would differ in their average abiotic conditions, despite identical experimental input of nutrients and organic matter at the metaecosystem scale. Similarly, we expected that dispersal-mediated alterations in abundances of species with large ecosystem effects would alter abiotic conditions and ecosystem functions. The directions of these effects, however, are difficult to predict for complex, multi-trophic communities because of opposing effects of organisms from different trophic levels (e.g., positive/ negative effects of primary producers/degraders on organic matter) and because of differential effects on multiple ecosystem processes (e.g., uptake of inorganic nutrients vs. production of organic matter by primary producers).

\section{Methods \\ Experimental design and setup}

In an outdoor mesocosm experiment, we studied the interactive effects of nutrient heterogeneity and ecosystem flux within a metaecosystem. We created a $2 \times 2$ factorial block design (Appendix S2: Fig. S1) by crossing 
two different nutrient loading regimes (homogenous (Homo) and heterogeneous (Hetero) nutrient loading) with two different ecosystem flux regimes (alive and dead ecosystem flux). Different ecosystem flux regimes were established in order to investigate how the flux of living organisms, organic material and nutrients, in contrast to the flux of only organic material and nutrients, affects community and ecosystem properties. Previous studies have tested metaecosystem theory by comparing treatments with and without input of nutrients or organic material into ecosystems (Pace et al. 2004, Graham et al. 2006, Jones and Lennon 2015). Our experiment, however, was designed to contrast metacommunity and metaecosystem dynamics, and test if changes in abiotic conditions might be mediated by the flux, establishment, and ecosystem effects of living organisms. Since our focus was not on contrasting unconnected and connected ecosystems, we did not include a treatment without the flux of nutrients and materials into our experimental design. Each metaecosystem replicate consisted of three $300 \mathrm{~L}$ tanks $(\varnothing=64 \mathrm{~cm}, \mathrm{~h}=100 \mathrm{~cm})$, which were connected by biweekly manual ecosystem flux. The four treatment combinations were replicated in 5 blocks, resulting in 20 metaecosystems. The mesocosm experiment ran for 20 weeks (May-September 2012) at the aquatic research institute Eawag in Kastanienbaum (Switzerland).

Mesocosms were initially filled with water from the nearby Lake Lucerne. All tanks additionally received equal quantities of water and the associated organisms (bacteria, phytoplankton, and zooplankton) from Lake Alpnach and Lake Rot prior to the start of the experiment. The different nutrient conditions were established through biweekly additions of phosphorus $(\mathrm{P})$, nitrogen $(\mathrm{N})$ and dissolved organic carbon (DOC). Within homogenous metaecosystems, each mesocosm received the same amounts of $\mathrm{P}, \mathrm{N}$, and DOC, while in heterogeneous metaecosystems, we established a high nutrient (HN), a low nutrient (L), as well as a high DOC mesocosm (Appendix S1: Table S1, Appendix S2: Fig. S1). The HN mesocosms received ten times as much $\mathrm{N}$ and $\mathrm{P}$ as both the $\mathrm{L}$ and the DOC mesocosms. $\mathrm{N}$ and $\mathrm{P}$ were provided as $\mathrm{NaNO}_{3}$ and $\mathrm{Na}_{2} \mathrm{HPO}_{4}$, respectively. The DOC mesocosms additionally received higher quantities of DOC leachate compared to the other mesocosms. While the loading of DOC also increased the loading of $\mathrm{P}$ and $\mathrm{N}$ (Appendix S1: Table S1), average loadings were equal for all metaecosystems. DOC leachate was produced by soaking standard garden peat for several weeks and then filtering the leachate through a $0.5 \mathrm{~mm}$ mesh prior to the addition to the mesocosms. At the metaecosystem scale, homogeneous and heterogeneous metaecosystems received the same amount of nutrients and DOC (Appendix S1: Table S1). To initiate the different nutrient conditions, each mesocosm received twice as much nutrients and DOC as added during the biweekly additions.

To establish different ecosystem flux regimes (alive vs. dead), equal quantities of water ( $1 \mathrm{~L}$ ) were sampled every second week from each tank within a metaecosystem (prior to the nutrient additions), mixed, and then redistributed in equal amounts $(300 \mathrm{~mL})$ to each mesocosm, either with or without living organisms. To kill the organisms within the dispersed water of the dead flux treatment, we autoclaved the water before redistributing it between tanks. We acknowledge that autoclaving the exchanged water might have mineralized organic matter into more easily available forms of nutrients, but we think that because of the low dispersal rate $(0.1 \%$ every other week) this effect was probably negligible given the levels of nutrients and organic matter in the mesocosms.

\section{Mesocosm sampling}

Over the course of the 20-week experiment, various biological, physical, chemical, and ecosystem functioning parameters were sampled and measured on a regular basis (Appendix S1: Table S2). We performed 10 biweekly samplings of algal biomass (Chlorophyll a), dissolved organic carbon (DOC) concentration and composition, as well as bacterial cell densities and cell size distributions. At the end of the experiment, we sampled the bacterial, phytoplankton, and zooplankton community and measured several other parameters (Appendix S1: Table S2). The bacterial community was sampled by filtering mesocosm water onto $0.2 \mu \mathrm{m}$ polyethersulfone filters (Supor 200 Membrane Disc Filters), which were instantly frozen in liquid nitrogen and preserved at $-80^{\circ} \mathrm{C}$ until further processing. Phytoplankton was sampled by sampling $100 \mathrm{~mL}$ mesocosm water from the water column and preserved with Lugol's solution. Integrated zooplankton samples were taken from the water column at 6-8 randomly chosen locations to sample a total volume of $10 \mathrm{~L}$. Zooplankton samples were filtered through a $30 \mu \mathrm{m}$ net and stored frozen prior to analysis.

\section{Sample processing}

Chlorophyll a (Chl-a) concentrations were determined on a high performance liquid chromatograph (HPLC) both from periphyton grown on $50 \mathrm{~cm}^{2}$ strips of plastic and from water filtered on ashed GF/F filters (Whatman). From the filtrate, we measured DOC concentrations on a Shimadzu TOC-V CPH, DOC absorption spectra over the wavelengths of 190 to $800 \mathrm{~nm}$ on a spectrophotometer, and concentrations of phosphate $\left(\mathrm{PO}_{4}\right)$ and nitrogen (the sum of $\mathrm{NO}_{3}$ and $\mathrm{NO}_{2}$ ) on an autoanalyzer. To determine bacterial cell densities and cell size distributions, $40 \mathrm{~mL}$ of tank water were fixed with a filtersterilized solution of paraformaldehyde and glutaraldehyde $(0.01 \%$ and $0.1 \%$ final concentrations, $\mathrm{pH} 7$; Nizzetto et al. 2012), and counts were measured on a BD Accuri ${ }^{\mathrm{TM}}$ C6 Flowcytometer. Conductivity, $\mathrm{pH}$, and temperature were measured in situ at approximately $0.5 \mathrm{~m}$ water depth using portable water sensors. We measured the sedimentation rate by hanging a $50 \mathrm{~mL}$ Falcon tube into each tank and weighing the dried, 
deposited material after several weeks. We estimated primary productivity by measuring the $\mathrm{O}_{2}$ concentrations in each tank three times over the course of $24 \mathrm{~h}$ (morning - evening - morning) and calculated gross and net primary productivity (GPP, NPP) and respiration (Resp) at the whole ecosystem level. We also measured respiration rates (Resp16) at controlled temperature conditions in the lab using custom-built temperature controlled glass vials at $16^{\circ} \mathrm{C}$ and fiber-optic oxygen mini-sensors (FIBOX 3, PreSens). $\mathrm{O}_{2}$ concentrations were measured every $30 \mathrm{~s}$ over the course of six hours and $\mathrm{O}_{2}$ uptake rates were calculated per hour.

The bacterial community composition was analyzed using Illumina sequencing. DNA was extracted by enzymatic digestion and cetyltrimethyl ammonium bromide (CTAB) extraction using a modified protocol from Lliros et al. (2008). Illumina sequencing and sequence analysis was performed as described previously (Birtel et al. 2015). We used the V3 region of the 16S rRNA for the analysis of bacterial diversity. Phytoplankton and zooplankton diversity and abundances were determined by microscopy. Phytoplankton was identified to genus level whenever possible and counted with an inverted microscope. Zooplankton was identified to species level when possible and enumerated with a dissecting microscope. For cladocerans and copepods, the total sample volume (corresponding to $10 \mathrm{~L}$ tank water) was counted, while rotifers were enumerated in subsamples.

\section{Data analysis}

All data was analyzed using the statistical software R version 3.1.3 (R Core Team 2015). We used the packages nlme (version 3.1.120) and vegan (version 2.3.0) to calculate linear mixed effect models and redundancy analyses, respectively. The sequencing data of the bacterial communities was analyzed using the bioinformatics pipeline QIIME (Kuczynski et al. 2011) prior to analyses in $\mathrm{R}$, as described previously (Birtel et al. 2015). Sequences were clustered at a sequence similarity threshold of $97 \%$, and bacterial communities were rarefied to 300 reads per mesocosm (Birtel et al. 2015). Prior to statistical analyses, the data was averaged over the three tanks of each metaecosystem.

Time series of Chl-a, DOC, and bacterial abundance were analyzed with profile analysis (Fidell and Tabachnick 2006). Profile analysis includes a test for main effects of treatments across time (levels test) and a test for an interaction between treatments and time (parallelism test). Levels tests were computed using linear mixed models with week and ecosystem flux or nutrient loading as fixed factors and metacommunity as random factor. Parallelism tests were computed using a MANOVA on the differences between successive time points.

We estimated the effect size by quantifying log response ratios (LRR) (Gruner et al. 2008) of the nutrient loading $(\mathrm{N})$ and ecosystem flux manipulation $(\mathrm{F})$, as well as their interaction $(\mathrm{FxN})$ of the environmental data as well as total biomass/abundance of phytoplankton, bacteria, and zooplankton, and ecosystem functions:

$$
\begin{aligned}
& \mathrm{LRR}_{\mathrm{N}}=\left(\ln \mathrm{Y}_{\mathrm{D}-\mathrm{Ho}}+\ln \mathrm{Y}_{\mathrm{A}-\mathrm{Ho}}\right)-\left(\ln \mathrm{Y}_{\mathrm{D}-\mathrm{He}}+\ln \mathrm{Y}_{\mathrm{A}-\mathrm{He}}\right) \\
& \mathrm{LRR}_{\mathrm{F}}=\left(\ln \mathrm{Y}_{\mathrm{D}-\mathrm{He}}+\ln \mathrm{Y}_{\mathrm{D}-\mathrm{Ho}}\right)-\left(\ln \mathrm{Y}_{\mathrm{A}-\mathrm{He}}+\ln \mathrm{Y}_{\mathrm{A}-\mathrm{Ho}}\right) \\
& \mathrm{LRR}_{\mathrm{FxN}}=\left(\ln \mathrm{Y}_{\mathrm{D}-\mathrm{Ho}}+\ln \mathrm{Y}_{\mathrm{A}-\mathrm{He}}\right)-\left(\ln \mathrm{Y}_{\mathrm{A}-\mathrm{Ho}}+\ln \mathrm{Y}_{\mathrm{D}-\mathrm{He}}\right)
\end{aligned}
$$

where $\mathrm{Y}$ is the mean response value among metaecosystems at the end of the experiment $\left(\mathrm{Y}_{\mathrm{D}-\mathrm{Ho}}\right.$ : average of metaecosystems with dead flux and homogenous nutrient loading; $\mathrm{Y}_{\mathrm{A}-\mathrm{Ho}}$ : average of metaecosystems with alive flux and homogenous nutrient loading; $\mathrm{Y}_{\mathrm{D}-\mathrm{He}}$ : average of metaecosystems with dead flux and heterogeneous nutrient loading; $\mathrm{Y}_{\mathrm{A}-\mathrm{He}}$ : average of metaecosystems with alive flux and heterogeneous nutrient loading). In order to compare the importance of treatments on different response variables, we standardized $\mathrm{Y}$ to a mean of zero and a standard deviation of one, after taking account of the block structure. The significance of the effects for the mean and coefficient of variation of the response variables was tested using linear mixed effect models with block as a random factor. Total abundances of phyto-, and zooplankton, and Chl-a of phytoplankton and periphyton were log-transformed prior to computing linear mixed effect models.

As a measure of local and regional species diversity, we calculated average $\alpha$ - and $\gamma$-diversity for each metaecosystem. Average $\alpha$-diversity (richness) was calculated as the number of OTUs for each mesocosm and then averaged across metaecosystems, and $\gamma$-diversity as the number of OTUs within each metaecosystem. We calculated average jaccard dissimilarity for each metaecosystem based on the presence-absence data as a measure of $\beta$-diversity. Bacterial diversity was averaged over 100 rarefactions to 300 reads. Treatment effects on diversity were determined using linear mixed effect models with block as a random factor. The species composition of bacteria, phytoplankton, and zooplankton communities was furthermore analyzed for treatment effects using distance based redundancy analysis (db-RDA) of both Hellinger-transformed abundance and presence-absence data and run with random block effect. $P$-values were calculated for the model attributes using 10,000 random permutations.

\section{RESUlTS}

\section{Ecosystem dynamics through time}

Over the course of the experiment, Chl-a, DOC, and bacterial numbers responded differently to both the spatial distribution of nutrient loading and the nature of ecosystem flux (Fig. 1; Appendix S1: Table S3). Metaecosystems with flux of living organisms had significantly higher levels of Chl-a than metaecosystems without flux of organisms, while the nutrient loading regime did not affect average metaecosystem Chl-a concentrations (Fig. 1A, B; Appendix S1: Table S3). The positive effect of live flux on Chl-a was particularly pronounced in $\mathrm{HN}$ 
Ecosystem flux
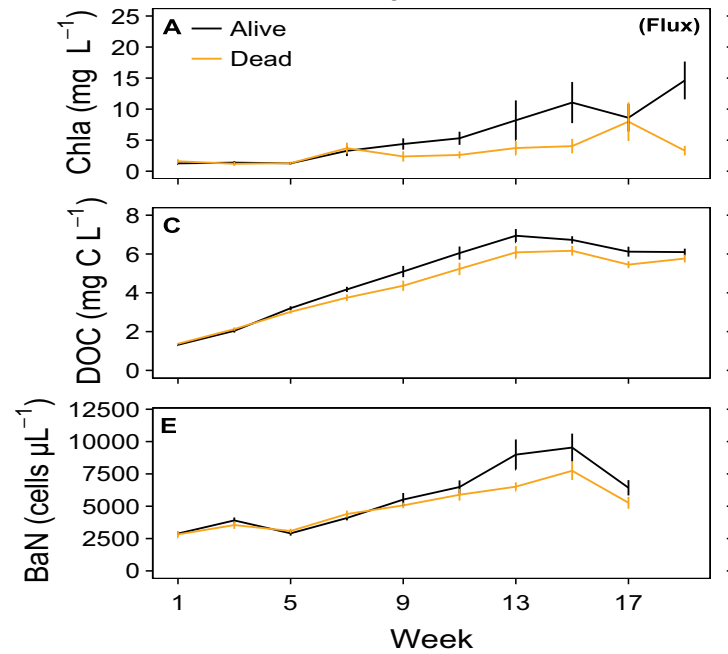
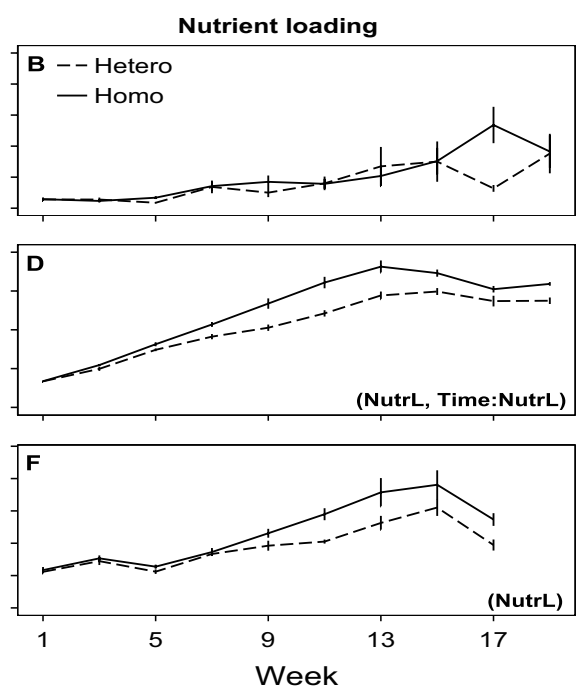

FIG. 1. Time-series plot of mean chlorophyll-a (Chla), DOC, and number of bacteria (BaN). Inserts in brackets denote significant effects of ecosystem flux (Flux), nutrient loading (NutrL), and significant interactions of treatments with time, as determined by profile analysis. A, C, E: data aggregated by ecosystem flux treatment (alive - dead); B, D, F: data aggregated by nutrient loading treatment (homogeneous - heterogeneous); values are mean $\pm \mathrm{SE}, N=10$. [Colour figure can be viewed at wileyonlinelibrary.com]

tanks (Appendix S2: Fig. S2A; levels test: $P=0.01$ ). The concentration of DOC and the number of bacteria were higher in homogeneous than in heterogeneous metaecosystems, but unaffected by ecosystem flux (Fig. 1C-F; Appendix S1: Table S3). At the scale of local patches, however, live flux increased DOC in HN tanks (Appendix S2: Fig. S2E; levels test: $P=0.05)$.

\section{Ecosystem properties at the end of the experiment}

Even though all replicated metaecosystems received identical total amounts of nutrients and DOC over the course of the 20-week experiment, at the end of the experiment the average environmental conditions differed between the homogeneous and heterogeneous loading regimes and the spatial heterogeneity of environmental conditions varied among treatments (Fig. 2; Appendix S1: Table S4). For example, conductivity and nitrate were higher in heterogeneous than in homogeneous metaecosystems, while $\mathrm{pH}$ and the concentration of DOC were higher in homogeneous than in heterogeneous metaecosystems. However, manipulating the living vs. non-living components of ecosystem flux had no effect on the abiotic components of the metaecosystems.

Across trophic levels, the treatment effects on average metaecosystem biomass or total abundance differed (Fig. 2A; Appendix S1: Table S4). For example, phytoplankton biomass (i.e., Chlorophyll-a) increased in the live ecosystem flux treatment, and the biomass of periphyton was higher in homogeneous than heterogeneous metaecosystems, but the total abundances of zooplankton and of bacteria did not vary among treatments.

Although average ecosystem functions (NPP, GPP, respiration) were unaffected by treatments (Fig. 2A;
Appendix S1: Table S4), spatial heterogeneity in nutrient loading increased the spatial variability of various environmental parameters and ecosystem functions (Fig. 2B; Appendix S1: Table S4), and the flux of living organisms increased spatial variability of Chlorophyll-a (Fig. 2B; Appendix S1: Table S4).

\section{Diversity and community composition}

Ecosystem flux, nutrient loading, and their interaction affected the spatial structure of biodiversity, but responses varied among trophic groups (Fig. 3; Appendix S1: Table S5), with greatest observed effects on small organisms (bacteria and phytoplankton). Both average $\alpha$ - and $\gamma$-diversity of the bacterial community were higher in metaecosystems with flux of live organisms (Fig. 3A+D), but mean community dissimilarity ( $\beta$-diversity) did not differ significantly among treatments (Fig. 3G). Live flux increased average $\alpha$-diversity of phytoplankton in heterogeneous metaecosystems, but had no effect in homogeneous metaecosystems (Fig. 3B). Both live flux and heterogeneity of nutrient loading resulted in an increase in phytoplankton $\gamma$-diversity (Fig. 3E), and interactively influenced $\beta$-diversity (Fig. 3H). Diversity of zooplankton communities was unaffected by treatments at any spatial scale. At the scale of local patches, nutrient identity and ecosystem flux affected phytoplankton diversity, but had no effect on the diversity of bacteria and zooplankton (Appendix S1: Table S6, Appendix S2: Fig. S3).

Treatments had strong effects on the community composition of bacteria and phytoplankton (db-RDA; Appendix S1: Table S7). The bacterial community composition was significantly affected by ecosystem flux and nutrient loading regimes when comparing species 

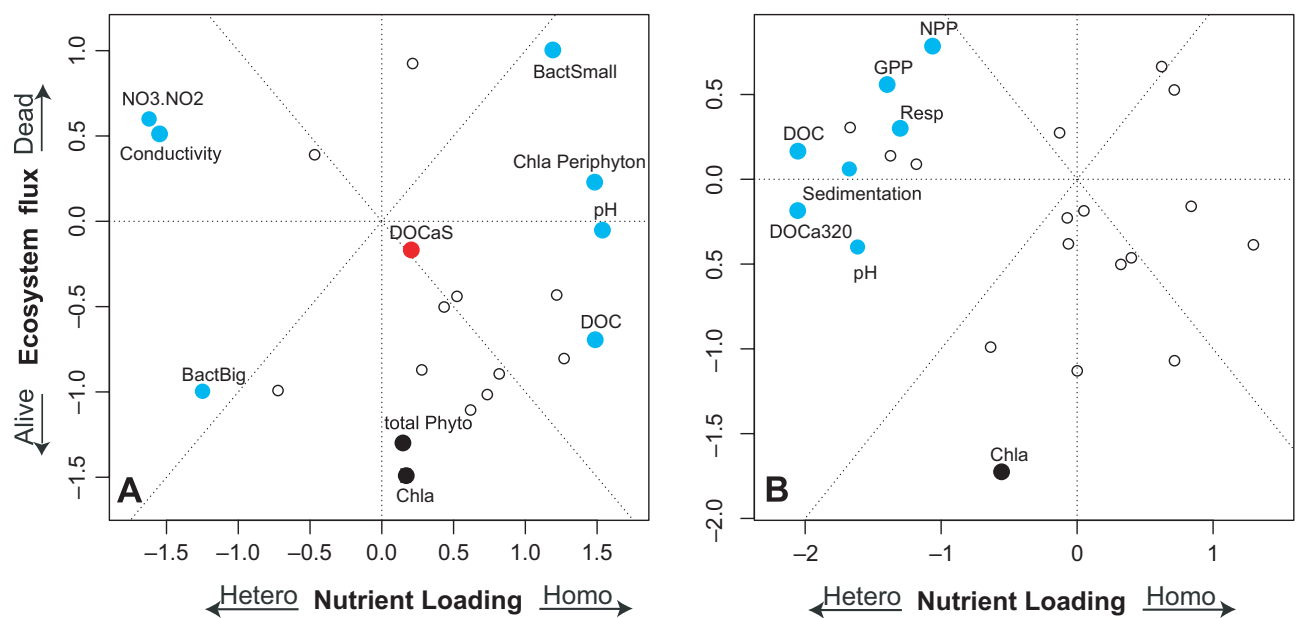

FIG. 2. Effect size (log response ratio LRR) and corresponding linear mixed effect (LME) results of ecosystem properties. Parameters with significant LME statistics displayed by colored, labeled dot. Blue dot: significant nutrient loading effect, black dot: significant ecosystem flux effect, red dot: significant interaction. A: LRR/LME results of metaecosystem mean values, B: LRR/LME results of metaecosystem coefficient of variation. Chla: Chlorophyll-a; DOC: dissolved organic carbon; DOCaS: DOC spectral slope; DOCa320: DOC absorption at $320 \mathrm{~nm}$, GPP: gross primary productivity; NPP: net primary productivity; Resp: respiration. [Colour figure can be viewed at wileyonlinelibrary.com]

identities, and by nutrient loading when comparing species abundances (Fig. 4A). Nutrient loading had significant effects on the community composition of the phytoplankton community based on species identities, but no effect based on abundances (Fig. 4B). The community composition of zooplankton was not significantly different among treatments (Fig. 4C). Nutrient identity, i.e., patch conditions (HN, L, DOC, Homo), affected the community composition at all trophic levels (Appendix S1: Table S8, Appendix S2: Figs. S4, S5).

\section{Discussion}

Our metaecosystem experiment tested for interactive effects of ecosystem flux and spatial heterogeneity of local environmental conditions on multiple aspects of aquatic ecosystems, including abiotic conditions and ecosystem functions, as well as the biomass, diversity, and composition of phytoplankton, bacteria, and zooplankton. We found that these three groups of organisms were differentially affected by the flux of living organisms; an outcome that can be explored using metacommunity theory. However, we also found that communities responded differently to our experimental manipulations of the environment, and that the resulting communities differentially modified local abiotic environmental conditions. Furthermore, such reciprocal interactions between biotic and abiotic compartments can be influenced by the flux of living organisms. Overall, we suggest that quantifying the diversity and composition of communities (i.e., metacommunity approach) alongside abiotic dynamics can provide novel insights into metaecosystem dynamics.

The observed patterns of phytoplankton diversity in our experiment generally follow predictions from the source-sink model of metacommunity theory (Mouquet and Loreau 2003). In heterogeneous metaecosystems, flux of living organisms resulted in an increase in average $\alpha$-diversity and in a decline in $\beta$-diversity, but had no effect in homogeneous metaecosystems (Fig. 3). One possibility is that live flux increased local richness in heterogeneous metaecosystems by maintaining inferior competitors in unfavorable habitats. For source-sink effects to operate, different environmental conditions have to result in different competitive hierarchies (Matthiessen et al. 2010). Hence, source-sink dynamics assume that metacommunities are structured by niche-based, deterministic processes. Accordingly, environmental heterogeneity increased $\gamma$-diversity, and the different patch conditions resulted in different community composition (Appendix S2: Figs. S4, S5), underlining the importance of deterministic processes in structuring the phytoplankton communities. An alternative possibility to such source-sink effects is that dispersal-mediated trophic interactions can contribute to the positive effects of live flux in heterogeneous metaecosystems (see below).

The flux of living organisms increased local diversity of bacteria, irrespective of environmental heterogeneity (Fig. 3A). In metacommunities with homogeneous environments, source-sink effects cannot operate (Leibold et al. 2004, Mouquet et al. 2006), but dispersal can affect diversity by rescuing species from dispersal limitation or stochastic extinctions (Leibold et al. 2004, Vellend 2010). The observed positive effect of live flux on bacterial diversity in homogeneous metacommunities could thus be due to live flux interacting with such stochastic processes. The pattern of beta diversity, with local communities sharing only few species regardless of heterogeneity (Fig. 3G), also suggests that ecological drift at least partly determined community structure (Chase and Myers 2011). However, contrasting local environmental 

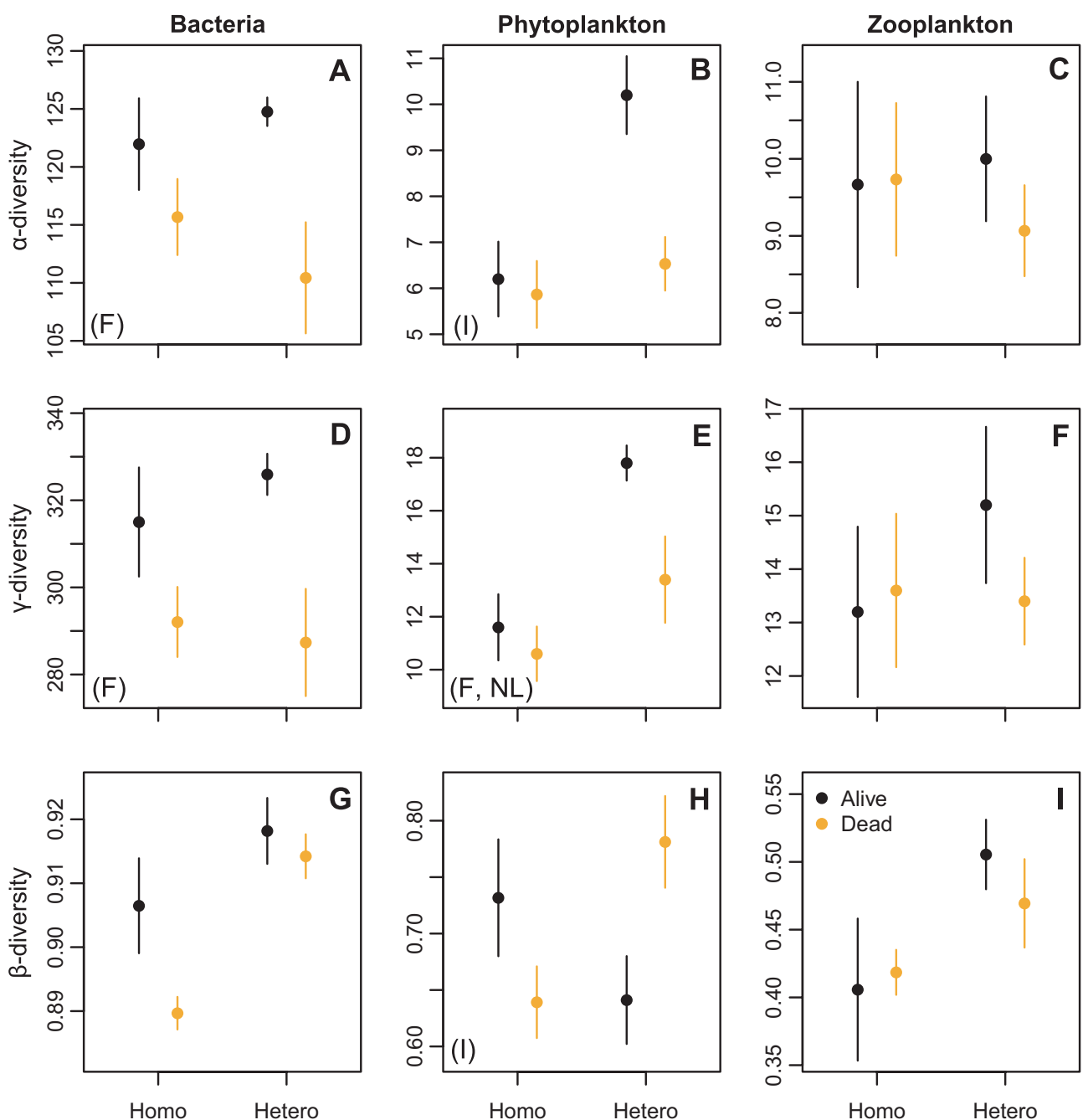

FIG. 3. Diversity estimates of the bacterial, phytoplankton, and zooplankton communities. Letters in brackets indicate significant LME statistics, (F): significant ecosystem flux effect; (NL): significant nutrient loading effect; (I): significant interaction. A-C: average metaecosystem $\alpha$-diversity, D-F: metaecosystem $\gamma$-diversity, $\mathrm{G}-\mathrm{I}$ : average metaecosystem Jaccard dissimilarity ( $\beta$ diversity). Mean $\pm \mathrm{SE}, N=5$. [Colour figure can be viewed at wileyonlinelibrary.com]

conditions explained variation in community composition, suggesting that deterministic processes were also important drivers of the bacterial community. It is well known, that both deterministic and stochastic processes can simultaneously influence communities (Adler et al. 2007, Vergnon et al. 2009, Chase and Myers 2011). For example, the importance of drift depends on community size and may thus be greater for rare than for abundant taxa (Hu et al. 2006), and may be more important within functional groups (Hubbell 2005). Previous observational studies have found that stochastic processes can be important in structuring bacterial communities (Östman et al. 2010, Farjalla et al. 2012, Soininen et al. 2013), while others found local environmental conditions to be the primary drivers of bacterial communities (Beisner et al. 2006, Lindström et al. 2010, De Bie et al. 2012, Hanson et al. 2012, Souffreau et al. 2015). A number of experimental studies found effects of dispersal on bacterial diversity (Declerck et al. 2013, Souffreau et al. 2014, Berga et al. 2015). As with these previous studies, our results suggest that the outcome of community composition reflects a balance of stochastic and niche-based processes, with the relative importance being context-dependent.

Zooplankton diversity was unaffected both by environmental heterogeneity and by live flux (Fig. 3). Metacommunity theory suggests that for dispersal to have a strong effect on diversity, a niche-based or a stochastic process (or both) must generate community differentiation. In metacommunities governed by nichebased processes, the effect of dispersal is expected to be highest at an intermediate level of heterogeneity (Mouquet et al. 2006). If local communities are highly similar because of low environmental heterogeneity, dispersal among communities will have only little effect on diversity, while strong species sorting can preclude effects of dispersal in metacommunities with high heterogeneity (Mouquet 

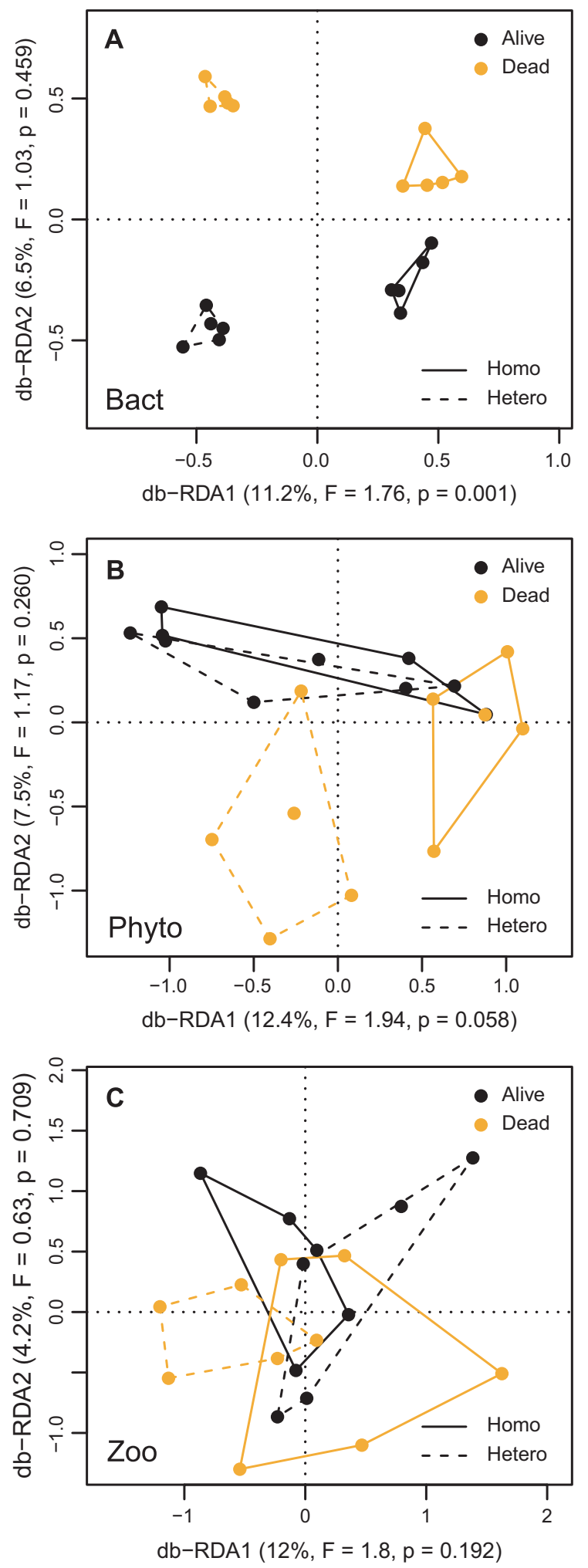

FIG. 4. db-RDA testing for effects of Ecosystem flux $\times$ Nutrient Loading on hellinger-transformed abundance data. (A) Bacteria, (B) Phytoplankton, (C) Zooplankton. [Colour figure can be viewed at wileyonlinelibrary.com] et al. 2006). In our experiment, zooplankton community composition differed among patch types (Appendix S2: Fig. S4C), indicating niche-based community differentiation. However, these differences in community composition were probably not strong enough for live flux to act as a structuring force on diversity. Ecological drift could also cause differentiation, but either local extinction dynamics might have been too slow or the inoculation of identical communities in local patches might have reduced the importance of dispersal limitation compared to natural metaecosystems.

The different effects of live flux among the three groups of organisms could be due to different processes governing community assembly. Previous observational studies found higher importance of stochastic processes in bacteria than in phyto- and zooplankton (Östman et al. 2010, Farjalla et al. 2012, Soininen et al. 2013), presumably because of higher plasticity in bacteria resulting in higher equivalence of taxa. Stochastic processes are predicted to be particularly important for organisms with small population sizes (Hu et al. 2006), and although bacteria are characterized by large community sizes, most taxa are rare (Sogin et al. 2006). Because of dispersal interacting with stochastic and deterministic processes (Vellend 2010), different relative importance of ecological drift vs. niche-based processes could be one possible explanation for the different effects of flux and heterogeneity on bacteria, phyto- and zooplankton observed in our experiment. Differences in dispersal ability between different groups of organisms are an alternative reason for differential effects of dispersal (De Bie et al. 2012), yet in our experiment the dispersal rate was the same for all three groups of organisms.

Differences in the effect of live flux could also be partly explained by different methods for determining community composition, and particularly the resolution of rare species. Detecting the relative importance of stochasticity and deterministic processes is an ongoing challenge in both comparative and experimental studies (Chase and Myers 2011, Vellend et al. 2014). Comparative studies vary in their support for stochasticity, and this might reflect differences in how communities are quantified among and within studies (Farjalla et al. 2012, Soininen et al. 2013). This is also true of experimental studies with multiple community types (Verreydt et al. 2012, Declerck et al. 2013). When comparing our results with those of observational studies, which find the importance of dispersal to increase with organism size (Beisner et al. 2006, De Bie et al. 2012), it is surprising that we did find effects of live flux on bacterial and phytoplankton communities, but not on zooplankton. Either performing a longer experiment, using a stronger environmental contrast for zooplankton (e.g., fish) (Howeth and Leibold 2010), or quantifying changes in genetic variation within zooplankton species (Pantel et al. 2011), might yield more insights into the processes governing community assembly in these groups. 
There is growing evidence for the importance of indirect effects of dispersal through dispersal-mediated trophic interactions (Verreydt et al. 2012), and we also see evidence for such indirect effects of dispersal in our experiment. Despite the lack of significant treatment effects on zooplankton diversity, effects on zooplankton community composition had strong effects on lower trophic levels. For example, most HN tanks without live flux were dominated by Daphnia, an efficient phytoplankton grazer, while HN tanks with live flux were dominated by rotifers (Appendix S2: Fig. S5C). These dispersal-mediated differences in the zooplankton community strongly affected phytoplankton biomass. Among all local patches, phytoplankton biomass was highest in $\mathrm{HN}$ tanks with live flux and lowest in HN tanks without live flux. Hence, the effects of flux on algal biomass were greater than those caused by our strong manipulation of nutrient loading (Appendix S2: Figs. S2, S6B). Given the low flux rate of only $0.1 \%$ every other week such a profound effect of flux is surprising and suggests that it was not due to flux of nutrients, but rather due to the effect of flux of living organisms on community composition. High relative abundances of Daphnia in HN tanks without live flux were likely the reason for the low phytoplankton biomass in these tanks. In HN tanks with live flux, the high algal biomass was likely caused by a dispersal-mediated change in the zooplankton community. Such dispersal-mediated trophic interactions might have also contributed to the observed increase of phytoplankton diversity in heterogeneous metaecosystems with live flux, and to the differential responses of phytoplankton and bacteria to the flux treatment.

Our experiment also revealed how the abiotic conditions of metaecosystems can be influenced by interactions between local ecosystem dynamics and the flux of organisms (or communities) with large ecosystem effects. Despite identical nutrient loading in all metaecosystems, homogeneous and heterogeneous metaecosystems diverged in numerous abiotic parameters (Fig. 2A). For example, DOC was consistently higher in homogeneous than heterogeneous metaecosystems (Fig. 1D), likely due to differential gains or losses of DOC that were mediated by mesocosm communities. Production of DOC by phytoplankton and periphyton was an important source of DOC in our metaecosystems, as illustrated by HN tanks that accumulated similarly high DOC concentrations as tanks with high experimental DOC input (Appendix S2: Figs. S2, S6A). Both the amount and the quality of algalderived DOC can be strongly affected by the nutrient status, physiological status, and composition of the algal community (Obernosterer and Herndl 1995, Giroldo and Vieira 2005, Carlson and Hansell 2015, Wear et al. 2015), and DOC released by algae can be nonlinearly related with primary production (Baines and Pace 1991). Because of such complex relationships between the algal community and algal-derived DOC, differential responses of the algal community to local nutrient loading in our experiment (Appendix S2: Figs. S2-S5) probably altered the local inputs of DOC. The observed differences in DOC could also be due to differential DOC degradation, caused by differences in bacterial abundance and/or community composition (Logue et al. 2016). However, the higher abundance of bacteria over the course of the experiment in homogeneous metaecosystems (Fig. 1F), suggests that reduced losses through bacterial degradation are an unlikely reason for higher DOC in homogeneous metaecosystems.

The ecosystem processes operating at local scales (e.g., DOC dynamics) can be influenced by dispersal-mediated alterations in community composition. In $\mathrm{HN}$ tanks, DOC increased in response to live flux (Appendix S2: Fig. S2E), suggesting that the dispersal-mediated increase in phytoplankton biomass fueled the DOC pool. Our experiment thus provides evidence that even in metaecosystems with only low direct flow of nutrients and material, habitat connectivity can affect the abiotic environment through a combination of dispersal-mediated alterations in community composition and modification of the environment by organisms. Biotic modification of ecosystem processes is not limited to the algae - DOC interaction, but is a driver of many ecosystem processes (Chapin et al. 1997, Jones et al. 1997, Largaespada et al. 2012, Schmitz et al. 2014). Also, nonlinear responses of communities to the abiotic environment and nonlinear effects of communities on ecosystem processes have been found for a variety of systems (Groffman et al. 2006, Lu et al. 2011, Klemmer et al. 2012, Vivanco et al. 2015). The metaecosystem perspective allows the integration of such interactions between biotic and abiotic ecosystem components into a framework of spatially structured ecosystems (Massol et al. 2011). Metaecosystem models suggest that local ecosystems can contribute unevenly to regional dynamics if they harbor species that play key roles (Mouquet et al. 2013). Our experiment suggests that species-specific modification of the environment and nonlinear relationships between ecosystem components can result in such disproportionate contributions of local ecosystems to metaecosystem dynamics. Hence, even subtle changes in community composition in response to local environmental conditions or habitat connectivity can have profound effects on metaecosystem dynamics.

\section{ACKNOWLEDGMENTS}

We wish to state that RL and JB contributed equally to this work. We thank Dany Steiner and Doris Hohmann for help with setting up and sampling of the mesocosm experiment, Regula Illi for counting phytoplankton abundances, and JeanClaude Walser and the GDC Zurich for assistance with Illumina library preparation and sequencing. We thank Helmut Bürgmann for helping to develop the project and Marek Svitok for help with statistical analyses. We thank two anonymous reviewers who helped to improve the manuscript. Furthermore, we acknowledge the financial support of the SNF grant 31003A-125006: "Food-web and ecosystem responses to global change" and of the SNF grant 31003A-153464/1: "The eco-evolutionary dynamics of community assembly in aquatic ecosystems." 


\section{Literature Cited}

Adler, P. B., J. HilleRisLambers, and J. M. Levine. 2007. A niche for neutrality. Ecology Letters 10:95-104.

Amarasekare, P. 2003. Competitive coexistence in spatially structured environments: a synthesis. Ecology Letters 6:1109-1122.

Atwood, T. B., E. Hammill, H. S. Greig, P. Kratina, J. B. Shurin, D. S. Srivastava, and J. S. Richardson. 2013. Predator-induced reduction of freshwater carbon dioxide emissions. Nature Geoscience 6:191-194.

Baines, S. B., and M. L. Pace. 1991. The production of dissolved organic matter by phytoplankton and its importance to bacteria: patterns across marine and freshwater systems. Limnology and Oceanography 36:1078-1090.

Bardgett, R. D., and D. A. Wardle. 2003. Herbivore-mediated linkages between aboveground and belowground communities. Ecology 84:2258-2268.

Beisner, B. E., P. R. Peres-Neto, E. S. Lindström, A. Barnett, and M. L. Longhi. 2006. The role of environmental and spatial processes in structuring lake communities from bacteria to fish. Ecology 87:2985-2991.

Berga, M., O. Östman, E. S. Lindström, and S. Langenheder. 2015. Combined effects of zooplankton grazing and dispersal on the diversity and assembly mechanisms of bacterial metacommunities. Environmental Microbiology 17:2275-2287.

Birtel, J., J.-C. Walser, S. Pichon, H. Bürgmann, and B. Matthews. 2015. Estimating bacterial diversity for ecological studies: methods, metrics, and assumptions. PLoS ONE 10:e0125356.

Carlson, C. A., and D. A. Hansell. 2015. DOM sources, sinks, reactivity, and budgets. Pages 65-126 in D. A. Hansell and C. A. Carlson, editors. Biogeochemistry of marine dissolved organic matter. Academic Press, London, UK.

Chapin, F. S., B. H. Walker, R. J. Hobbs, D. U. Hooper, J. H. Lawton, O. E. Sala, and D. Tilman. 1997. Biotic control over the functioning of ecosystems. Science 277:500-504.

Chase, J. M., and J. A. Myers. 2011. Disentangling the importance of ecological niches from stochastic processes across scales. Philosophical Transactions of the Royal Society B 366:2351-2363.

Cherif, M., and M. Loreau. 2013. Plant - herbivore - decomposer stoichiometric mismatches and nutrient cycling in ecosystems. Proceedings of the Royal Society B-Biological Sciences 280:20122453.

De Bie, T., et al. 2012. Body size and dispersal mode as key traits determining metacommunity structure of aquatic organisms. Ecology Letters 15:740-747.

Declerck, S. A. J., C. Winter, J. B. Shurin, C. A. Suttle, and B. Matthews. 2013. Effects of patch connectivity and heterogeneity on metacommunity structure of planktonic bacteria and viruses. ISME Journal 7:533-542.

Farjalla, V. F., D. S. Srivastava, N. A. C. Marino, F. D. Azevedo, V. Dib, P. M. Lopes, A. S. Rosado, R. L. Bozelli, and F. A. Esteves. 2012. Ecological determinism increases with organism size. Ecology 93:1752-1759.

Fidell, L. S., and B. G. Tabachnick. 2006. Using multivariate statistics. Harper and Row, New York, New York, USA.

Gessner, M. O., C. M. Swan, C. K. Dang, B. G. McKie, R. D. Bardgett, D. H. Wall, and S. Haettenschwiler. 2010. Diversity meets decomposition. Trends in Ecology and Evolution 25: 372-380.

Giroldo, D., and A. A. H. Vieira. 2005. Polymeric and free sugars released by three phytoplanktonic species from a freshwater tropical eutrophic reservoir. Journal of Plankton Research 27:695-705.

Graham, M. D., R. D. Vinebrooke, and M. Turner. 2006. Coupling of boreal forests and lakes: effects of conifer pollen on littoral communities. Limnology and Oceanography 51: 1524-1529.

Gravel, D., F. Guichard, M. Loreau, and N. Mouquet. $2010 a$. Source and sink dynamics in meta-ecosystems. Ecology 91: $2172-2184$

Gravel, D., N. Mouquet, M. Loreau, and F. Guichard. $2010 b$. Patch dynamics, persistence, and species coexistence in metaecosystems. The American Naturalist 176:289-302.

Groffman, P., et al. 2006. Ecological thresholds: the key to successful environmental management or an important concept with no practical application? Ecosystems 9:1-13.

Gruner, D. S., et al. 2008. A cross-system synthesis of consumer and nutrient resource control on producer biomass. Ecology Letters 11:740-755.

Hanson, C. A., J. A. Fuhrman, M. C. Horner-Devine, and J. B. H. Martiny. 2012. Beyond biogeographic patterns: processes shaping the microbial landscape. Nature Reviews Microbiology 10:497-506.

Howeth, J. G., and M. A. Leibold. 2010. Species dispersal rates alter diversity and ecosystem stability in pond metacommunities. Ecology 91:2727-2741.

Hu, X.-S., F. He, and S. P. Hubbell. 2006. Neutral theory in macroecology and population genetics. Oikos 113:548-556.

Hubbell, S. P. 2005. Neutral theory in community ecology and the hypothesis of functional equivalence. Functional Ecology 19:166-172.

Jones, S. E., and J. T. Lennon. 2015. A test of the subsidystability hypothesis: the effects of terrestrial carbon in aquatic ecosystems. Ecology 96:1550-1560.

Jones, C. G., J. H. Lawton, and M. Shachak. 1997. Positive and negative effects of organisms as physical ecosystem engineers. Ecology 78:1946-1957.

Klemmer, A. J., S. A. Wissinger, H. S. Greig, and M. L. Ostrofsky. 2012. Nonlinear effects of consumer density on multiple ecosystem processes. Journal of Animal Ecology 81:770-780.

Kuczynski, J., J. Stombaugh, W. A. Walters, A. Gonzalez, J. G. Caporaso, and R. Knight. 2011. Using QIIME to analyze 16S rRNA gene sequences from microbial communities. Current Protocols in Bioinformatics 36:10.17.11-10.17.20.

Largaespada, C., F. Guichard, and P. Archambault. 2012. Meta-ecosystem engineering: nutrient fluxes reveal intraspecific and interspecific feedbacks in fragmented mussel beds. Ecology 93:324-333.

Leibold, M. A., et al. 2004. The metacommunity concept: a framework for multi-scale community ecology. Ecology Letters 7:601-613.

Lindström, E. S., X. M. Feng, W. Graneli, and E. S. Kritzberg. 2010. The interplay between bacterial community composition and the environment determining function of inland water bacteria. Limnology and Oceanography 55:2052-2060.

Lliros, M., E. O. Casamayor, and C. Borrego. 2008. High archaeal richness in the water column of a freshwater sulfurous karstic lake along an interannual study. FEMS Microbiology Ecology 66:331-342.

Logue, J. B., C. A. Stedmon, A. M. Kellerman, N. J. Nielsen, A. F. Andersson, H. Laudon, E. S. Lindstrom, and E. S. Kritzberg. 2016. Experimental insights into the importance of aquatic bacterial community composition to the degradation of dissolved organic matter. ISME Journal 10:533-545.

Loreau, M., N. Mouquet, and R. D. Holt. 2003. Metaecosystems: a theoretical framework for a spatial ecosystem ecology. Ecology Letters 6:673-679.

Lu, M., Y. Yang, Y. Luo, C. Fang, X. Zhou, J. Chen, X. Yang, and B. Li. 2011. Responses of ecosystem nitrogen cycle to nitrogen addition: a meta-analysis. New Phytologist 189: $1040-1050$ 
Marleau, J. N., F. Guichard, F. Mallard, and M. Loreau. 2010. Nutrient flows between ecosystems can destabilize simple food chains. Journal of Theoretical Biology 266: $162-174$.

Marleau, J. N., F. Guichard, and M. Loreau. 2014. Metaecosystem dynamics and functioning on finite spatial networks. Proceedings of the Royal Society B-Biological Sciences 281:20132094.

Massol, F., D. Gravel, N. Mouquet, M. W. Cadotte, T. Fukami, and M. A. Leibold. 2011. Linking community and ecosystem dynamics through spatial ecology. Ecology Letters 14: 313-323.

Matthiessen, B., E. Mielke, and U. Sommer. 2010. Dispersal decreases diversity in heterogeneous metacommunities by enhancing regional competition. Ecology 91:2022-2033.

Mouquet, N., and M. Loreau. 2003. Community patterns in source-sink metacommunities. The American Naturalist 162:544-557.

Mouquet, N., T. E. Miller, T. Daufresne, and J. M. Kneitel. 2006. Consequences of varying regional heterogeneity in source-sink metacommunities. Oikos 113:481-488.

Mouquet, N., D. Gravel, F. Massol, and V. Calcagno. 2013. Extending the concept of keystone species to communities and ecosystems. Ecology Letters 16:1-8.

Nizzetto, L., R. Gioia, J. Li, K. Borga, F. Pomati, R. Bettinetti, J. Dachs, and K. C. Jones. 2012. Biological pump control of the fate and distribution of hydrophobic organic pollutants in water and plankton. Environmental Science and Technology 46:3204-3211.

Obernosterer, I., and G. J. Herndl. 1995. Phytoplankton extracellular release and bacterial growth: dependence on the inorganic N: P ratio. Marine Ecology Progress Series 116:247-257.

Östman, O., S. Drakare, E. S. Kritzberg, S. Langenheder, J. B. Logue, and E. S. Lindstrom. 2010. Regional invariance among microbial communities. Ecology Letters 13:118-127.

Pace, M. L., J. J. Cole, S. R. Carpenter, J. F. Kitchell, J. R. Hodgson, M. C. Van de Bogert, D. L. Bade, E. S. Kritzberg, and D. Bastviken. 2004. Whole-lake carbon-13 additions reveal terrestrial support of aquatic food webs. Nature 427:240-243.

Pantel, J. H., M. A. Leibold, and T. E. Juenger. 2011. Population differentiation in Daphnia alters community assembly in experimental ponds. The American Naturalist 177:314-322.
R Core Team. 2015. R: A language and environment for statistical computing. R foundation for statistical computing. Vienna, Austria. http://www.R-project.org/

Schmitz, O. J., et al. 2014. Animating the carbon cycle. Ecosystems 17:344-359.

Sogin, M. L., H. G. Morrison, J. A. Huber, D. Mark Welch, S. M. Huse, P. R. Neal, J. M. Arrieta, and G. J. Herndl. 2006. Microbial diversity in the deep sea and the underexplored "rare biosphere". Proceedings of the National Academy of Sciences of the United States of America 103:12115-12120.

Soininen, J., J. J. Korhonen, and M. Luoto. 2013. Stochastic species distributions are driven by organism size. Ecology 94:660-670.

Souffreau, C., B. Pecceu, C. Denis, K. Rummens, and L. De Meester. 2014. An experimental analysis of species sorting and mass effects in freshwater bacterioplankton. Freshwater Biology 59:2081-2095.

Souffreau, C., et al. 2015. Environmental rather than spatial factors structure bacterioplankton communities in shallow lakes along a $>6000 \mathrm{~km}$ latitudinal gradient in South America. Environmental Microbiology 17:2336-2351.

Vellend, M. 2010. Conceptual synthesis in community ecology. Quarterly Review of Biology 85:183-206.

Vellend, M., et al. 2014. Assessing the relative importance of neutral stochasticity in ecological communities. Oikos 123: $1420-1430$

Vergnon, R., N. K. Dulvy, and R. P. Freckleton. 2009. Niches versus neutrality: uncovering the drivers of diversity in a species-rich community. Ecology Letters 12:1079-1090.

Verreydt, D., L. De Meester, E. Decaestecker, M. J. Villena, K. Van der Gucht, P. Vannormelingen, W. Vyverman, and S. A. J. Declerck. 2012. Dispersal-mediated trophic interactions can generate apparent patterns of dispersal limitation in aquatic metacommunities. Ecology Letters 15:218-226.

Vivanco, L., I. C. Irvine, and J. B. H. Martiny. 2015. Nonlinear responses in salt marsh functioning to increased nitrogen addition. Ecology 96:936-947.

Wear, E. K., C. A. Carlson, A. K. James, M. A. Brzezinski, L. A. Windecker, and C. E. Nelson. 2015. Synchronous shifts in dissolved organic carbon bioavailability and bacterial community responses over the course of an upwelling-driven phytoplankton bloom. Limnology and Oceanography 60: 657-677.

\section{SUPPORTING INFORMATION}

Additional supporting information may be found in the online version of this article at http://onlinelibrary.wiley.com/ doi/10.1002/ecy.1742/suppinfo 